\title{
Successful Conversion Surgery Following Chylous Ascites After Nivolumab for Advanced Gastric Cancer
}

\author{
SATOSHI TOYOTA ${ }^{1}$, HIROYUKI ORITA $^{1}$, YASURO FUKUYAMA ${ }^{1}$, \\ SAKI MOTOYOSHI ${ }^{1}$, SHOGO KAWANAMI ${ }^{1}$, SHOHEI MAEDA ${ }^{1}$, \\ ERINA KURAMITSU ${ }^{1}$, MICHIHIRO ICHIMANDA ${ }^{1}$, SATOKO NAGAMATSU ${ }^{1}$, \\ SHIGEYUKI NAGATA ${ }^{1}$, SEIICHIRO KAI ${ }^{1}$, DAISUKE KORENAGA ${ }^{1}$ and MASAKI MORI ${ }^{2}$ \\ ${ }^{1}$ Department of Surgery, Nakatsu Municipal Hospital, Nakatsu, Japan; \\ ${ }^{2}$ Department of Surgery and Science, Graduate School of Medical Sciences, Kyushu University, Fukuoka, Japan
}

\begin{abstract}
Case Report: A 75-year-old man who underwent nivolumab as the third-line chemotherapy for advanced gastric cancer had chylous ascites (CA) after the primary tumor shrank remarkably. Total parenteral nutrition and subcutaneous octreotide were initiated, and CA disappeared. Nivolumab was continued for a total of 23 courses. Computed tomography showed lymph node swelling; however, staging laparoscopy showed that peritoneal metastasis had disappeared. Therefore, conversion surgery and distal gastrectomy with $\mathrm{Dl}+$ dissection were performed. Results: The pathological diagnosis was type $I V$, poorly differentiated adenocarcinoma (por2) with signet ring cells, ypT2 (muscularis propria), without lymphatic or venous invasion, and no involvement of the proximal and distal margins. After the operation, no recurrence was observed over 7 months with no adjuvant chemotherapy. Conclusion: Nivolumab has the potential to lead to RO resection for patients with peritoneal carcinomatosis gastric cancer. To our knowledge, this is the first report of successful conversion surgery after nivolumab-related chylous ascites.
\end{abstract}

Nivolumab (Bristol-Myers Squibb, Ono Pharmaceutical), a monoclonal antibody to human $\mathrm{IgG} 4$ programmed cell death protein 1 (PD1)-blocking, has efficacy in a range of malignancies. Results from a randomized, double-blind, placebocontrolled, phase III trial (ONO-4538-12, ATTRACTION-2) (1) showed that nivolumab as a third-line chemotherapy prolonged the overall survival of patients with advanced gastric cancer

This article is freely accessible online.

Correspondence to: Satoshi Toyota, Department of Surgery, Nakatsu Municipal Hospital, Nakatsu, Oita, Japan. Tel: +81 979222480, Fax: +81 979222481, e-mail: sa-toyoda@nakatsu-hosp.jp

Key Words: Gastric cancer, peritoneal metastasis, nivolumab, chylous ascites, conversion surgery, pseudo progression.
(GC). On the other hand, immune-related adverse events such as interstitial pneumonitis, dysthyroidism, and type 1 diabetes have been reported (1). The activated immune system is assumed to cause these unique events; however, it is poorly understood. This report describes a case of successful conversion surgery after chylous ascites (CA) due to nivolumab as a third-line chemotherapy for advanced GC.

\section{Case Report}

A 75-year-old man was admitted to our hospital for the treatment of GC in January 2017. Preoperative endoscopy revealed an advanced GC (Borrmann's type 3 ) occupied the entire circumference of the antrum (Figure 1A and B).

Preoperative computed tomography (CT) revealed slight swelling of the lesser curvature lymph node and no distant metastasis (Figure 1C). Staging laparoscopy revealed peritoneal metastases (category 3), so curative resection was abandoned and the patient was started on first-line chemotherapy as S-1 plus oxaliplatin (SOX). After two courses, CT revealed a growing primary tumor (chemotherapy effect judgement: Progressive disease). The patient underwent gastrojejunostomy because of stenosis by the tumor, and second-line chemotherapy of paclitaxel plus ramucirumab was administered. After seven courses, although the primary tumor shrank slightly, the clinical condition worsened. The patient abandoned continuing secondline chemotherapy and was started on nivolumab $240 \mathrm{mg} /$ body as third-line chemotherapy in February 2018. After two courses, the primary tumor shrank (Figure 2B); however, he presented significant abdominal distension, and computed tomography (CT) showed massive ascites (Figure 2A). He was hospitalized, and cell-free and concentrated ascites reinfusion therapy (CART) was performed, which showed no malignant cells and milky ascites (Figure 2C) (triglyceride level $455 \mathrm{mg} / \mathrm{dl}$ ). Total parenteral nutrition and $300 \mu \mathrm{g} /$ day subcutaneous octreotide was initiated for the chylous ascites, and it diminished gradually. After 1 month, CART (each punctured ascites, 4,800, 4,400, 

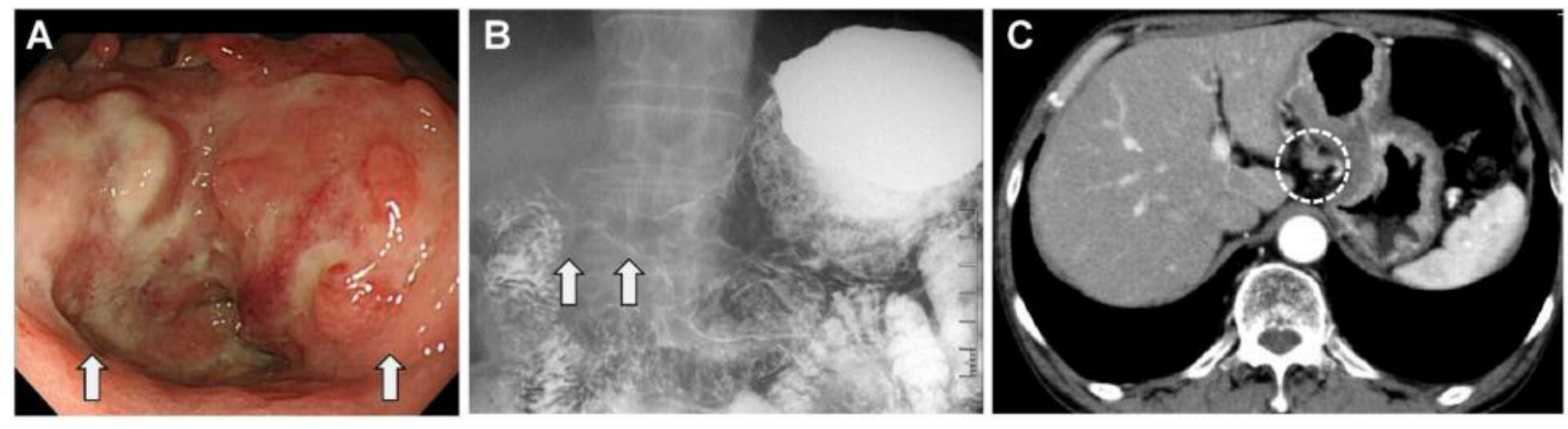

Figure 1. A: First examination of gastric endoscopy showed Borrmann's type 2 gastric cancer that occupied the entire circumference of the antrum (arrows). B: Upper gastrointestinal study revealed an irregularly constricted pyloric zone (arrows). C: First computed tomographic imaging revealed slight swelling at the lesser curvature lymph node (circle) but no distant metastasis.
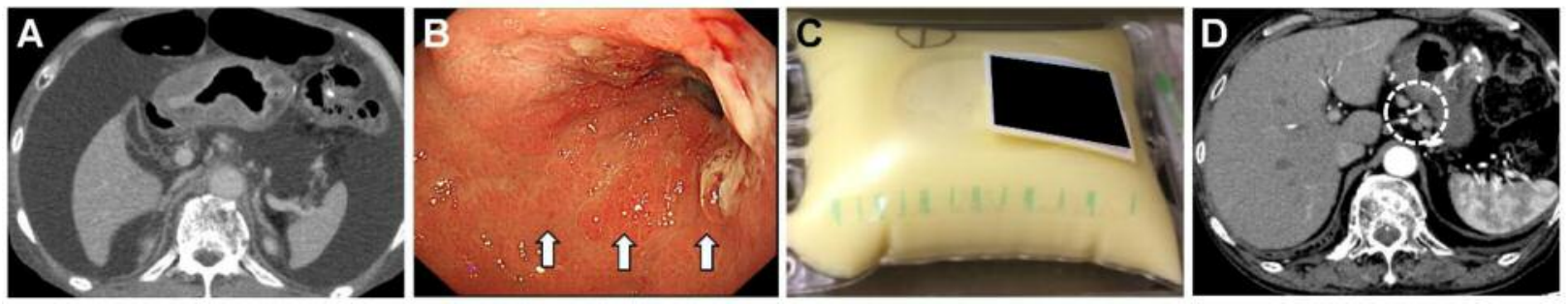

Figure 2. A: Computed tomographic imaging after the second course of nivolumab revealed massive ascites, showing primary lesion shrinkage. B: Gastric endoscopy after nivolumab showed that the primary lesion had shrunk (arrows). C: Punctured chylous ascites. D: Curvature lymph node swelling shown again by computed tomographic imaging (circle).

5,700 , and 2,500 ml) was performed four times, and there was no sign of chylorrhea (triglyceride level $105 \mathrm{mg} / \mathrm{dl}$ ), so the patient was discharged from the hospital. Nivolumab was continued for a total of 23 courses. Follow-up CT results showed lesser curvature lymph node swelling (Figure 2D); however, staging laparoscopy showed peritoneal metastases had disappeared. Therefore, distal gastrectomy with D1+ dissection was performed.

\section{Results}

The pathological diagnosis was type IV, poorly differentiated adenocarcinoma (por2) with signet ring cells, ypT2 (muscularis propria), without lymphatic $(0 / 32)$ or venous invasion, and no involvement of the proximal and distal margins. in the Japanese Classification of Gastric Carcinoma (2). After the operation, no recurrence was observed over 7 months with no adjuvant chemotherapy.

\section{Discussion}

To our knowledge, this is the first report of successful conversion surgery after nivolumab-related CA. Peritoneal carcinomatosis is the most frequent site of metastasis, occurring in $14-43 \%$ of patients with stage IV GC (3), and the survival of patients with stage IV GC, especially those with peritoneal carcinomatosis, is poor (4). However, Okabe et al. noted that the median survival time (MST) of some curatively (R0) resected patients after the disappearance of peritoneal dissemination treated with chemotherapy was longer than that of patients without surgery (MST: $43 v s .10 .3$ months) (5). Due to the development of new drugs and regimens, the number of cases of conversion surgery is increasing. Most are performed for patients with advanced GC who exhibited dramatic effects on first-line chemotherapy (3). Therefore, a successful R0 resection after third-line chemotherapy is valuable and provides significant hope for peritoneal carcinomatosis of GC.

In addition to nivolumab efficacy, some prudent assessments contributed to the successful outcome. Firstly, the patient was not abandoned at the third line despite the appearance of CA, a rare form of ascites characterized by a milky fluid that contains high levels of triglyceride (the current consensus utilizes a cutoff value of $>200 \mathrm{mg} / \mathrm{dl}$ as the criterion for diagnosis). Most CA are due to lymph leakage caused by malignancy progression, cirrhosis, infection, trauma and surgical intervention (6), and the 1-year mortality rate 
associated with CA due to malignancy progression is reported to be $90 \%$ (7). However, cytology of the ascites showed that malignant cells had disappeared; therefore, third-line chemotherapy of nivolumab was continued after treatment for CA. The mechanism of this CA was not clear, but Yanagihara et al. (8) reported a kidney cancer patient whose lung metastases shrank markedly after developing pleural effusion containing lymphocytes (68\%) after nivolumab therapy. Based on the report, although cytological analysis was not performed, it is possible that nivolumab attracts lymphocytes, and accumulated lymphocytes might attack or occult the lymph duct, leading to the development of CA. As another hypothesis, the molecular targeted drug lenvatinib (an inhibitor of vascular endothelial growth factor receptor-2 and -3 kinase), is reported to cause arterial bleeding in differentiated thyroid carcinoma (9). This is because of rapid tumor shrinkage tearing the feeding artery. In our case, tumor shrinkage was rapid, and the same destruction might have occurred in the lymph duct.

After 23 courses, follow-up CT showed lesser curvature lymph node swelling as if there were lymph node metastases. However, following the pathological diagnosis, all 32 examined lymph nodes were free of carcinoma cells, and this CT finding was possibly related to pseudo progression, a false appearance of disease progression upon imaging examination that occurs in approximately $10 \%$ of patients receiving immunotherapy (10). It is often difficult to distinguish from true progression. Some reports have stated that decreased serum tumor marker levels may be correlated with clinical response (11); however, the tumor markers carcinoembryonic antigen and carbohydrate antigen 19-9 were normal at all times. Clinical conditions are also found to be helpful for discrimination (11). In this case, the endoscopic findings showed that the primary lesion remained shrunken and that the patient's clinical condition was good, therefore we thought that it might be pseudo progression.

In conclusion, a patient with stage IV GC with peritoneal carcinomatosis underwent successful conversion surgery due to nivolumab, third-line chemotherapy. Immune checkpoint inhibitors, such as nivolumab, may have the potential to facilitate R0 resection for patients with peritoneal carcinomatosis of GC but the unique phenomena of CA and pseudo progression can confuse judgement of cancer staging and therapy evaluation. Therefore, prudent assessment from various viewpoints is required.

\section{Conflicts of Interest}

The Authors declare they have no conflicts of interest.

\section{Authors' Contributions}

Study conception: ST. Data collection: ST. Writing: ST. Critical review and revision: HO, YF, SM, SK, SM, EK, MI, SN, SN, SK, DK, MM. Final approval of the article: All Authors. Accountability for all aspects of the work: All Authors.

\section{References}

1 Kang YK, Boku N, Satoh T, Ryu MH, Chao Y, Kato K, Chung HC, Chen JS, Muro K, Kang WK, Yeh KH, Yoshikawa T, Oh SC, Bai LY, Tamura T, Lee KW, Hamamoto Y, Kim JG, Chin K, Oh DY, Minashi K, Cho JY, Tsuda M and Chen LT: Nivolumab in patients with advanced gastric or gastro-oesophageal junction cancer refractory to, or intolerant of, at least two previous chemotherapy regimens (ono-4538-12, attraction-2): A randomised, double-blind, placebo-controlled, phase 3 trial. Lancet 390(10111): 2461-2471, 2017. PMID: 28993052. DOI: 10.1016/s0140-6736(17)31827-5

2 Japanese Gastric Cancer Association: Japanese classification of gastric carcinoma: 3rd English edition. Gastric Cancer 14: 101112, 2011. DOI: 10.1007/s10120-011-0041-5

3 Zurleni $\mathrm{T}$, Gjoni E, Altomare $\mathrm{M}$ and Rausei $\mathrm{S}$ : Conversion surgery for gastric cancer patients: A review. World J Gastrointest Oncol 10(11): 398-409, 2018. PMID: 30487951. DOI: $10.4251 /$ wjgo.v10.i11.398

4 Chau I, Norman AR, Cunningham D, Waters JS, Oates J and Ross PJ: Multivariate prognostic factor analysis in locally advanced and metastatic esophago-gastric cancer-pooled analysis from three multicenter, randomized, controlled trials using individual patient data. J Clin Oncol 22(12): 2395-2403, 2004. PMID: 15197201. DOI: 10.1200/jco.2004.08.154

5 Okabe H, Ueda S, Obama K, Hosogi H and Sakai Y: Induction chemotherapy with S-1 plus cisplatin followed by surgery for treatment of gastric cancer with peritoneal dissemination. Ann Surg Oncol 16(12): 3227-3236, 2009. PMID: 19777180. DOI: 10.1245/s10434-009-0706-Z

6 Ilhan E, Demir U, Alemdar A, Ureyen O, Eryavuz Y and Mihmanli M: Management of high-output chylous ascites after D2-lymphadenectomy in patients with gastric cancer: A multicenter study. J Gastrointest Oncol 7(3): 420-425, 2016. PMID: 27284475. DOI: $10.21037 /$ jgo.2016.02.03

7 Vasko JS and Tapper RI: The surgical significance of chylous ascites. Arch Surg 95(3): 355-368, 1967. PMID: 4166570. DOI: 10.1001/archsurg.1967.01330150031006

8 Yanagihara T, Tanaka K, Ota K, Kashiwagi E, Takeuchi A, Tatsugami K, Eto M, Nakanishi Y and Okamoto I: Tumorinfiltrating lymphocyte-mediated pleuritis followed by marked shrinkage of metastatic kidney cancer of the chest wall during nivolumab treatment. Ann Oncol 28(8): 2038-2039, 2017. PMID: 28459940. DOI: 10.1093/annonc/mdx214

9 Suyama K, Murakami D, Fujiwara S, Takeshita T, Sueta A, Inao T, Yamamoto-Ibusuki M, Yamamoto Y, Shiraishi S-Y and Iwase $\mathrm{H}$ : Massive arterial bleeding after lenvatinib therapy for thyroid cancer. Int J Cancer Clin Res 3(6): 2378-3419, 2016. DOI: 10.23937/2378-3419/3/6/1074

10 Wolchok JD, Hoos A, O’Day S, Weber JS, Hamid O, Lebbé C, Maio M, Binder M, Bohnsack O, Nichol G, Humphrey R and Hodi FS: Guidelines for the evaluation of immune therapy activity in solid tumors: Immune-related response criteria. Clin Cancer Res 15: 7412-7420, 2009. PMID: 19934295. DOI: 10.1158/1078-0432.CCR-09-1624

11 Passler M, Taube ET, Sehouli J and Pietzner K: Pseudo-or real progression? An ovarian cancer patient under nivolumab: A case report. World J Clin Oncol 10(7): 247-255, 2019. PMID: 31396474. DOI: 10.5306/wjco.v10.i7.247

Received November 5, 2019

Revised November 12, 2019 Accepted November 13, 2019 